\title{
Factors that predict clinical outcome after colectomy for fulminant Clostridium difficile colitis
}

\author{
I. Michael Leitman*, Rahul Narang, Jorys Martinez, Benjamin Abbadessa, Dimitrios Avgerinos, \\ Erez Dayan, Sebastiano Cassaro
}

Department of Surgery, Albert Einstein College of Medicine, Beth Israel Medical Center, New York, USA

Email: ․ㅡleitman@chpnet.org

Received 4 March 2012; revised 5 April 2012; accepted 6 April 2012

\section{ABSTRACT}

Background: Historically, the incidence of $C$. difficileassociated colitis has increased significantly over the last decade. Of these patients, approximately $10 \%$ $\mathbf{2 0 \%}$ will ultimately require colectomy due to fulminant disease and progression to toxic colitis. Despite operative intervention, the mortality for this population remains high (35\% - 80\%). This study evaluates for preoperative risk factors for mortality. Methods: Retrospective medical record review of 47 patients who underwent emergent colectomy for fulminant $C$. difficile colitis over a five-year period at three teaching hospitals in New York City. Results: Forty-seven patients with Clostridium difficile colitis underwent colectomy from January 2003 to December 2008. The mean age was 66 years with 31 (67\%) male and 16 (34\%) female. The 30-day mortality was $34 \%$ (16/47 patients). Thirty-eight (81\%) underwent subtotal colectomy, six (13\%) Hartmann resection, two (4\%) right hemicolectomy, and one (2\%) underwent left colectomy. Univariate analysis showed that age (71.6 vs. 67.5 years; $p=0.402$ ), white blood cell count $\left(35,500\right.$ vs. $\left.27,700 / \mathrm{mm}^{3} ; p=0.271\right)$, and use of vasopressors in the pre-operative period $p=0.440$ ) were not statistically predictive of postoperative mortality. Conversely, preoperative serum lactate level (4.3 vs. $2.1 \mathrm{mmol} / \mathrm{L} ; \mathrm{p}=\mathbf{0 . 0 0 9}$ ) was statistically greater in the postoperative mortality group. Conclusion: In the setting of fulminant $C$. difficile colitis, serum lactate levels can help predict postoperative outcome following emergent colectomy and should be closely followed to facilitate the decision to proceed with surgery.

Keywords: Colectomy; Surgery; Clostridium difficile; Colitis; Outcome; Sepsis; Lactate; Diarrhea; Hospital Acquired Infection

*Corresponding author.

\section{INTRODUCTION}

The exposure of colonic anaerobic gut flora to antibiotics creates a microenvironment that favors the colonization and proliferation of gram-positive anaerobic bacillus Clostridium difficile (C. difficile). The production of enterotoxin A and cytotoxin B induce an inflammatory process that causes a wide spectrum of disease severity and symptomatology, ranging from asymptomatic colonization and mild diarrhea to severe, fulminant colitis and toxic megacolon associated with increased morbidity and mortality. The increase in morbidity and mortality rates associated with this disease has been previously reported [1].

Historically, the incidence of $C$. difficile-associated colitis has increased significantly over the last decades in the U.S. hospitals. Of these patients, approximately $10 \%$ - $20 \%$ will end up requiring colectomy due to fulminant disease progression to toxic megacolon.

Hospital acquired infectious diarrheal illness is a major cause of morbidity and mortality.

However, despite operative management, the mortality of the patient population remains high (35\% - 80\%) most often due to sepsis and multiple organ dysfunction [2-4]. Patients older than 80 years of age are reported to have a mortality rate of 86 percent. This study investigates the factors that predict mortality and clinical outcomes in patients with Clostridium difficile colitis that undergo operative management.

\section{PATIENTS AND METHODS}

After approval from the Institutional Review Board of our hospitals, a retrospective cohort review study was conducted of patients with $C$. difficile colitis that underwent colectomy in three major teaching hospitals in New York City, spanning a 5-year period.

The following data were analyzed: demographics, past medical and surgical history, clinical presentation, laboratory and radiological results, use of vasopressor support, type of operation, pathology report, post-operative 
complications, and 30-day mortality.

The Fisher exact test was used for analysis of data and determination of p-value. The test was used to determine whether the proportions of those falling into each category differ by group. We performed an exact one-tailed and two-tailed p-values for a given frequency table.

All patients had a positive stool Clostridium difficile cytotoxin assay (enzyme-linked immunosorbent assays for toxin A and B, Techlab Enteric Diagnostics, Blacksburg, VA). All patients were in rooms with two or more patients. Patients underwent operative management due to fulminant colitis (defined in cases where colectomy was performed on an emergency basis and subsequent pathologic analysis identified pseudo-membranous colitis).

The extent of surgery (subtotal or segmental colectomy) was determined by the operating surgeon, based upon pre-operative colonoscopy or CT scan.

\section{RESULTS}

Forty-seven patients with Clostridium difficile colitis underwent colectomy from January 2003 to December 2008. The mean age was 66 years, and there were 31 (66\%) male and 17 (34\%) female patients (Table 1).

All patients had co-morbid disease, such as end stage renal disease (10), hypertension (35), coronary artery disease (28), chronic obstructive pulmonary disease (6), diabetes (19), and inflammatory bowel disease (10). The majority of the patients (93\%) received antibiotics within last 30 days before developing $C$. difficile colitis.

A C. difficile toxin test was ordered on hospitalized patients that had frequent loose stools, abdominal pain, fever, and/or nausea. An enzyme linked immunoabsorbant assay (ELISA) for toxin A and B was performed on all patients. There was a $100 \%$ rate of isolation of toxin A and/or B through cytotoxin assay from stool sample preoperatively. 39 patients had computed tomography
(CT) scans of the abdomen and pelvis, with evaluation of colonic wall thickening, and ancillary findings of pericolonic stranding and ascites.

Of all patients, thirty-eight patients (81\%) received subtotal colectomy; six (13\%) received a Hartmann's procedure, two (4\%) received right hemicolectomy, and one (2\%) patient received left hemicolectomy (Table 2).

The 30-day mortality rate was 34\% (16 out of 47 patients). Univariate analysis showed that age (71.56 vs. 67.46 years; $p=0.402)$, white blood cell count $(35,300$ vs. $27,700 / \mathrm{mm}^{3} ; \mathrm{p}=0.271$ ), and use of vasopressors in the pre and post-operative period $(p=0.440$ ) were not significantly different between patients that died and the ones that survived (Table 3). Conversely, preoperative serum lactate level (4.3 vs. $2.1 \mathrm{mmol} / \mathrm{L} ; \mathrm{p}=0.009$ ) was significantly increased in the mortality group.

\section{DISCUSSION}

The incidence of Clostridium difficile — associated colitis has increased significantly over the last decades. Subsequently, this has also led to an increase in patients requiring colectomy due to the disease progression to fulminant toxic megacolon. Several reports have suggested that $C$. difficile - associated diarrhea is evolving into a more severe disease; however, the link between the organism's potential virulence factors and severity in clinical presentation has not been established [5-8].

The clinical presentation of diarrhea and inflammation are results of the toxins produced by $C$. difficile. Most well characterized are exterotoxin (toxin A) and cytotoxin (toxin B). Toxins A and B are glucosyl transferases, while toxin A induces actin depolymerization by a mechanism correlated with a decrease in ADP-ribosylation of GTP-binding Rho proteins [9].

Gram staining for the organism has gram-positive cells that have optimum growth on blood agar at human body temperatures in the absence of oxygen $[10,11]$. Under

Table 1. Patient population demographics and characteristics.

\begin{tabular}{|c|c|c|c|c|}
\hline Characteristics & All Patients $(n=47)$ & Mortality Group (n = 16) & Survivor Group (n = 31) & $\mathrm{p}$-Value \\
\hline Mean age (year) & 66 & 71.56 & 67.46 & \\
\hline Gender $(\mathrm{M} / \mathrm{F})$ & $31 / 16$ & $12 / 6$ & $19 / 10$ & 0.1255 \\
\hline Ulcerative colitis & 7 & 1 & 6 & 0.3956 \\
\hline Crohn's disease & 3 & 0 & 3 & 0.5412 \\
\hline Diabetes mellitus & 19 & 5 & 14 & 0.5316 \\
\hline COPD & 6 & 3 & 4 & 0.999 \\
\hline CAD & 28 & 9 & 19 & 0.7631 \\
\hline Hypertension & 35 & 11 & 24 & 0.7252 \\
\hline End-stage renal disease (ESRD) & 10 & 2 & 8 & 0.4568 \\
\hline Received antibiotics within last 30 days & 44 & 15 & 29 & 0.999 \\
\hline
\end{tabular}

$\mathrm{COPD}=$ chronic obstructive pulmonary disease, $\mathrm{CAD}=$ coronary artery disease. 
Table 2. Type of operation performed.

\begin{tabular}{lcccc}
\hline Types of Operation & $\begin{array}{c}\text { Mortality } \\
\text { Group }\end{array}$ & $\begin{array}{c}\text { Survivor Total Patients } \\
\text { Group }\end{array}$ & (\%) & p-Value \\
\hline Subtotal colectomy & 15 & 23 & $38(81 \%)$ & 0.138 \\
Hartman's & 1 & 5 & $6(13 \%)$ & 0.6484 \\
Right hemicolectomy & 0 & 2 & $2(4 \%)$ & 0.5412 \\
Left hemicolectomy & 0 & 1 & $1(2 \%)$ & 0.999 \\
Total patients (\%) & $16(34 \%)$ & $31(66 \%)$ & $47(100 \%)$ & \\
\hline
\end{tabular}

stressed condition, the bacteria forms spore. These acidresistant spores ingested by patient are able to pass through the stomach and germinate in the colon upon exposure to bile acids and multiply.

Endoscopy and stool cytotoxin titers have both falsenegative rates, making the accurate diagnosis $C$. difficile colitis preoperatively difficult at times $[7,12,13]$. For this study, all patients had a positive stool $C$. difficile cytotoxin assay and underwent surgery on an emergency basis for toxic megacolon. The subsequent pathologic analysis was consistent with pseudo-membranous colitis, confirming the disease process.

Studies have reported the mortality range from fulminant $C$. difficile colitis requiring surgical management to range from $33 \%$ - $80 \%[7,14,15]$. This risk of post-operative complications and mortality is observed to be even higher in the elderly population, especially over the age of 80 years, who have an additive increased mortality rate of $>20 \%$ [16]. The mean age of our patients was 66years and 30-day mortality rate was 34\%.

The association between exposure to antibiotics and $C$. difficile-associated diarrhea is well known. Greater than $90 \%$ of our patients received antibiotics within 30 days of developing fulminant colitis. It is a possibility that the use of new formulary of antibiotics may promote the formation of more resistant and virulent strains of $C$. difficile. As a result, leading to more frequent failure of medical management and hence, a higher incidence of fulminant disease. An example includes the epidemic of a clindamycin resistant strain among patients who received the antibiotic [9].

Accepted surgical practices include administration of perioperative antibiotics for patients undergoing procedures to prevent surgical site infections. As high as 35\% of patient who develop fulminant $C$. difficile colitis have only history of perioperative antibiotics exposure and is presumed to be the cause of development of pseudomembranous colitis [17].

The majority of the patients in our study were male, as observed in many other studies. In comparing the demographics and characteristics of our study population, a minority of patients had a history of inflammatory bowel disease (IBD). Good prognosis with high survival rate was noted within this group of patients. Historically, colectomy for fulminant $C$. difficile colitis is associated with high mortality among patients with IBD. As recommended by the literature, most of our patients received subtotal colectomy with end ileostomy. However, a minority of patients underwent segmental colectomy (Hartmann's, right or left hemicolectomy).

Among the spectrum of medical and surgical management of $C$. difficile colitis, there is often the dilemma of conservative management of antibiotics and serial abdominal examination versus the alternative of surgical management. Retrospective analysis have indicated that late surgical intervention for fulminant disease is associated with higher postoperative mortality, however, the guideline for patient selection for operative management is not clearly defined [18]. High clinical suspicion with indications for need for vasopressors support, or signs and symptoms of end-organ damage are strong indications requiring operative management. Osman and coworkers reported that patients that fail to respond to maximum medical therapy and develop three of the following should be evaluated for possible colectomy: abdominal pain, abdominal distension, localized tenderness, pyrexia $>38^{\circ} \mathrm{C}$, and tachycardia $>100$ beats per minute. In addition to the above, if the patient is above 65 years old and develops four of the following, they should be considered for an emergency colectomy: WBC $>16 \times$ 10(9)/l, lactate > $2.2 \mathrm{mmol} / \mathrm{l}$, albumin $<30 \mathrm{~g} / \mathrm{l}$, blood pressure $<90 \mathrm{~mm} \mathrm{Hg}$, CT/endoscopy evidence of severe colitis in spite of maximum antibiotic treatment [19].

In the patients who underwent surgery for fulminant $C$. difficile colitis, the predictive postoperative mortality rate remains to be high despite the advancement in understanding of the disease process and aggressive clinical management, including antibiotics (metronidazole, vancomycin, vancomycin enemas) and earlier surgical management. Several factors were evaluated in the patients who underwent surgical management. These preoperative factors were used to determine the correlation be

Table 3. Predictors of motrality.

\begin{tabular}{lcccc}
\hline \multicolumn{1}{c}{ Variable } & Mean Value in Mortality Group & Mean Value in Survivor Group & p-Value & 95\% Confidence Interval of Difference \\
\hline Age (years) & 71.56 & 67.46 & 0.402 & $-9.4-11.4$ \\
WBC $\left(/ \mathrm{mm}^{3}\right.$ ) & 35,300 & 27,700 & 0.271 & $-2.7-1.9$ \\
Serum Lactate (mmol/L) & 4.3 & 2.1 & 0.009 & $-4.15--0.75$ \\
Pressors (no. of patients) & 11 & 18 & 0.44 & $-1.8-9.1$ \\
\hline
\end{tabular}


tween postoperative clinical outcomes. We found that preoperative serum lactate level was the only statistically significant laboratory value in predicting postoperative mortality (Table 3). Others have published similar association between serum lactate levels and mortality in patients undergoing emergent colectomy for fulminant $C$. difficile colitis [20-22]. Analysis showed that age and white blood cell count was not significantly different in patients that survived and died. Studies have reported that preoperative vasopressor requirement is predictive of mortality, correlating with severe sepsis, multisystem organ failure and death $[5,23,24]$. Interestingly, we found that use of vasopressors did not play a significant role in predicting mortality. Making the decision to proceed with emergent colectomy remains a challenge to the practitioner.

As we continue to understand the factors that dictate the patient outcome, further research is needed to identify resistant virulent strains of this disease and its application to the clinical scenario. Studies in advance understanding in the treatment of this disease include evaluating the first-line treatment of $C$. difficile colitis, where the use of antibiotics, particularly metronidazole appears to be cost effective in regards to hospital stay and recurrence of the disease [25].

The epidemiology of the disease is important to identify the patients susceptible. Recent analysis shows that the spectrum of patients is shifting toward the outpatient setting, with most cases are acquired in the community or in nursing home facility [26].

Within the hospital, transmission of this virulent $C$. difficile strain could have occurred from a multitude of factors other than antibiotic therapy, such as colonized healthcare workers and other infected patients. The toxin transmitted from person to person by the fecal-oral route. The organism forms large numbers of heat-resistant spores, which are not eradicated by alcohol-based hand cleansers. It has been reported that patients with private single bedrooms have a lower incidence of $C$. difficileassociated diarrhea compared with double-bedded room [27]. The majority of rooms in our institutions have multiple beds (as many as four beds to one room) with shared lavatories, which may be an important factor in its transmissibility.

In summary, fulminant $C$. difficile colitis has very high death rate and rapid diagnosis and treatment is crucial to a positive outcome. Patients who are unresponsive to medical treatment require early surgical management. Further research is needed to improve medical treatments, including inhibitors of the inflammatory process of disease.

\section{CONCLUSION}

Recent research concerning the preoperative and postop- erative predictors of mortality following colectomy in the setting of fulminant $C$. difficile colitis has shown that earlier operative intervention trends toward improved morbidity and 30-day mortality [7]. However, there has yet to be a consensus concerning the preoperative variables most predictive of post operative mortality. Based on this study, we believe that in the setting of fulminant C. difficile colitis, serum lactate levels can help predict clinical outcome and should be closely followed and considered in the clinical algorithm used to determine the timeliness of operative intervention.

\section{REFERENCES}

[1] Johnson, S., Samore, M.H., Farrow, K.A., et al. (1999) Epidemics of diarrhea caused by a clindamycin-resistant strain of Clostridium difficile in four hospitals. The New England Journal of Medicine, 341, 1645-1651. doi:10.1056/NEJM199911253412203

[2] Fekety, R. (1997) Guidelines for the diagnosis and management of Clostridium difficile-Associated diarrhea and colitis. The American Journal of Gastroenterology, 92, 739-750.

[3] Grundfest-Broniatowski, S., Quader, M., Alexander, F., Walsh, R.M., Lavery, I. and Milsom, J. (1996) Clostridium difficile colitis in the critically ill. Diseases of the Colon \& Rectum, 39, 619-623. doi:10.1007/BF02056938

[4] Synnott, K., Mealy, K., Merry, C., Kyne, L., Keane, C. and Quill, R. (1998) Timing of surgery for fulminating pseudo-membranous colitis. British Journal of Surgery, 85, 229-231. doi:10.1046/j.1365-2168.1998.00519.x

[5] Lamontagne, F., Labbé, A.C., Haeck, O., et al. (2007) Impact of emergency colectomy on survival of patients with fulminant Clostridium difficile colitis during an epidemic caused by a hypervirulent strain. Annals of Surgery, 245, 267-272. doi:10.1097/01.sla.0000236628.79550.e5

[6] Byrn, J.C., et al. (2008) Predictors of mortality after colectomy for fulminant Clostridium difficile colitis. Archives of Surgery, 143, 150-154. doi:10.1001/archsurg.2007.46

[7] Sailhamer, E.A., et al. (2009) Fulminant Clostridium difficile colitis: Patterns of care and predictors of mortality. Archives of Surgery, 144, 433-439. doi:10.1001/archsurg.2009.51

[8] Perera, A.D., et al. (2010) Colectomy for fulminant Clostridium difficile colitis: Predictors of mortality. The American Journal of Surgery, 76, 418-421.

[9] Just, I., Selzer, J., Von Eichel-Streiber, C. and Aktories, K. (1995) The enterotoxin from Clostridium difficile (ToxA) monoglucosylates the Rho proteins. Journal of clinical investigation, 95, 1026-1031. doi:10.1172/JCI117747

[10] Kurian, A., et al. (2011) In-hospital and 6-month mortality rates after open elective vs open emergent colectomy in patients older than 80 years. Diseases of the Colon \& Rectum, 54, 467-471. doi:10.1007/DCR.0b013e3182060904 
[11] Morris, A.M., Jobe, B.A., Stoney, M., Sheppard, B.C., Deveney, C.W. and Deveney, K.E. (2002) Clostridium difficile colitis: An increasingly aggressive iatrogenic disease? Archives of Surgery, 137, 1096-1100. doi:10.1001/archsurg.137.10.1096

[12] Dallal, R.M., Harbrecht, B.G., Boujoukas, A.J., et al. (2002) Fulminant Clostridium difficile: An underappreciated and increasing cause of death and complications. Annals of Surgery, 235, 363-372. doi:10.1097/00000658-200203000-00008

[13] Muto, C.A., Pokrywka, M., Shutt, K., et al. (2005) A large outbreak of Clostridium difficile-Associated disease with an unexpected proportion of deaths and colectomies at a teaching hospital following increased fluoroquinolone use. Infection Control and Hospital Epidemiology, 26, 273-280. doi:10.1086/502539

[14] Klipfel, A., Schein, M., Fahoum, B. and Wise, L. (2000) Acute abdomen and Clostridium difficile colitis: Still a lethal combination. Digestive Surgery, 17, 160-163. doi:10.1159/000018821

[15] Samore, M.H., Venkataraman, L., DeGirolami, P.C., Arbeit, R.D. and Karchmer, A.W. (1996) Clinical and molecular epidemiology of sporadic and clustered cases of nosocomial Clostridium difficile diarrhea. American Journal of Medicine, 100, 32-40. doi:10.1016/S0002-9343(96)90008-X

[16] Ryan, K.J. and Ray, C.G. (2004) Sherris medical microbiology. 4th Edition, McGraw Hill, New York.

[17] Al-Abed, Y.A., Gray, E.A. and Rothnie, N.D. (2010) Outcomes of emergency colectomy for fulminant Clostridium difficile colitis. Surgeon, 8, 330-333. doi:10.1016/j.surge.2010.06.003

[18] Butala, P. and Divino, C.M. (2010) Surgical aspects of fulminant Clostridium difficile colitis. The American Journal of Surgery, 200, 131-135.

\section{doi:10.1016/j.amjsurg.2009.07.040}

[19] Pepin, J., Vo, T.T., Boutros, M., et al. (2009) Risk factors for mortality following emergency colectomy for fulminant Clostridium difficile infection. Diseases of the Colon \& Rectum, 52, 400-405. doi:10.1007/DCR.0b013e31819a69aa

[20] Pepin, J., Valiquette, L., Alary, M.E., et al. (2004) Clostridium difficile-Associated diarrhea in a region of Quebec from 1991 to 2003: A changing pattern of disease severity. Canadian Medical Association Journal, 171, 466-472. doi:10.1503/cmaj.1041104

[21] Osman, K.A., Ahmed, M.H., Hamad, M.A. and Mathur, D. (2011) Emergency colectomy for fulminant Clostridium difficile colitis: Striking the right balance. Scandinavian Journal of Gastroenterology, 46, 1222-1227. doi:10.3109/00365521.2011.605469

[22] Olivas, A.D., Umanskiy, K., Zuckerbraun, B. and Alverdy, J.C. (2010) Avoiding colectomy during surgical management of fulminant Clostridium difficile colitis. Surgical Infections (Larchmt), 11, 299-305. doi:10.1089/sur.2010.026

[23] Thomas, K.L., Holmes, K.R., Jackson, B.R., et al. (2007) A cost comparison of metronidazole and vancomycin treatment of Clostridium difficile-Associated diarrhea. The American Journal of Gastroenterology, 102, 268.

[24] Kumar, V., Yoselevitz, S. and Dutta, S.K. (2007) Clostridium difficile colitis: A shift from a traditional hospital acquired infection to a community and nursing homebased disease. The American Journal of Gastroenterology, 102, 266.

[25] Stewart, D.B. and Hollenbeak, C.S. (2011) Clostridium difficile colitis: Factors associated with outcome and assessment of mortality at a national level. Journal of Gastrointestinal Surgery, 15, 1548-1555. doi:10.1007/s11605-011-1615-6 